\title{
Review of: "The Drosophila TMEM184B ortholog Tmep ensures proper locomotion by restraining ectopic firing at the neuromuscular junction"
}

Beatriz Blanco Redondo ${ }^{1}$

1 Universität Leipzig

Potential competing interests: The author(s) declared that no potential competing interests exist.

It is a very elegant study using genetics as well as behavioural, electrophysiological and immunohistochemical analysis.

The authors show novelty in the study by creating new fly models which can help to elucidate mechanisms in how Tmep (TMEM184B in mice) works.

They could nicely recapitulate the phenotype in flies and characterised its expression pattern.

I only have minor comments on the manuscript:

- $59 \%$ identical protein level between human and fly is not too much

- It would be nice to see how WTs behave in figure 1C

- I would not show the EJP numbers in 4 different graphs if the statistics do not show significance and state that NMJs impair locomotion activity with this data 